\title{
Pérdidas Económicas Causadas por Nacobbus aberrans y Globodera spp. en el Cultivo de la Papa en Bolivia
}

\author{
Javier Franco ${ }^{1}$, Janett Ramos ${ }^{2}$, Rolando Oros ${ }^{2}$, \\ Gladys Maín ${ }^{2}$, Noel Ortuño ${ }^{3}$
}

\begin{abstract}
Resumen
La información disponible sobre la distribución (incidencia) y pérdidas en el rendimiento (severidad) causadas por Nacobbus aberrans y Globodera spp. en la producción de papa fue analizada para estimar las pérdidas económicas que estos fitoparásitos ocasionan al cultivo en Bolivia. Los resultados obtenidos por extrapolación de áreas cultivadas, incidencia, severidad de daño y precio de venta de los tubérculos han permitido estimar que las pérdidas económicas en el valor bruto de la producción de papa en Bolivia alcanzan a US\$52 millones con $N$. aberrans y US\$16 millones con Globodera spp. La especie dominante en este último género es $G$. pallida en relación a $G$. rostochiensis. Ambas especies se encuentran más frecuentemente entre los 3,500 a los 4,000 msnm a diferencia de $N$. aberrans que se le encuentra mayormente entre los 3,000 a 4,000 msnm.
\end{abstract}

Palabras claves adicionales: incidencia, severidad, pérdidas económicas.

Aceptado para publicación: octubre 1998.

1 Ph.D., Departamento de Nematología, Programa de Investigación de la Papa (PROINPA), Casilla 4285, Cochabamba, Bolivia.

2 Ing. Agr., Departamento de Nematología, Programa de Investigación de la Papa (PROINPA), Casilla 4285, Cochabamba, Bolivia.

3 M.Sc., Departamento de Nematología, Programa de Investigación de la Papa (PROINPA), Casilla 4285, Cochabamba, Bolivia 


\section{Economic Losses Caused by Nacobbus aberrans and Globodera spp. to the Potato Crop in Bolivia}

\section{Summary}

Information available on the distribution (incidence) and yield losses (severity) caused by Nacobbus aberrans and Globodera spp. in potato production was analyzed to estímate the economic losses that these phytoparasites cause to the potato crop in Bolivia. The results obtained by the extrapolation of cultivated areas, incidence, severity damage, and price of the tubers, allowed to estímate the economic loss of the gross value of potato praduction in Bolivia, reaching US\$52 million with $N$. aberrans and US\$16 million with Globodera spp. The dominating species in this genus is $G$. pallida in relation to $G$. rostochiensis. Both species are most frequently found between 3,500 masl and 4,000 masl, whereas $N$. aberrans is found primarily between 3,000 to 4,000 masl.

Additional keywords: incidence, severity, gross value loss.

\section{Introducción}

Entre los cultivos andinos, la papa es el producto más importante en Bolivia porque constituye el alimento fundamental en la dieta diaria de la población, tanto en cantidad como en calidad. Se produce en diferentes techos ecológicos del país desde 1,600 hasta los 4,500 msnm, comprendiendo siete departamentos de los nueve que tiene el país.

Considerando la estructura de minifundio de la agricultura tradicional boliviana, alrededor de 180,000 unidades de producción familiar cultivan alrededor de 0.76 ha de papa por unidad agropecuaria, esto significa que un $28 \%$ de los campesinos del país producen papa. Este porcentaje es considerablemente mayor en el caso de Oruro, Potosí y Chuquisaca. Adicionalmente el cultivo está generando más de 134,000 fuentes de trabajo directo, pero incluyendo actividades indirectas se puede estimar que entre 200 a 250 mil personas se encuentran involucradas en diversas actividades de la producción. 
En 1963-65, la papa representaba casi el 45\% del total de productos agrícolas, pero esa participación se redujo gradualmente hasta un $17.4 \%$ en el trienio $1985-87$, para luego ascender nuevamente al 23.3\% en el trienio 1990-92. Sin embargo, en términos del valor bruto de la producción, la papa mantiene una posición importante sobre el total del valor de productos agrícolas (US\$206'156,161). Es así que, considerando un grupo de ocho cultivos principales en la agricultura boliviana y adoptando como factor común los precios internacionales en 1994, la papa en términos de valor absoluto sigue siendo muy importante (42).

Los rendimientos varían notablemente, sin embargo, el promedio nacional estimado es de $4 \mathrm{t} / \mathrm{ha}$, el cual constituye uno de los más bajos en el mundo. Este rendimiento promedio tan bajo, se debería a que la papa es un producto altamente sensible a los factores climáticos (sequía y heladas) y también a problemas fitosanitarios. Algunos de estos problemas fitosanitarios se han tornado endémicos porque se han difundido muy rápidamente en muchas de las zonas productoras del país, lo cual se traduce en la pérdida de considerables superficies productoras de papa.

Entre los problemas fitosanitarios, Nacobbus aberrans y Globodera spp. son dos de los nemátodos fitoparásitos que causan los mayores perjuicios económicos en la producción del cultivo de papa de la zona andina, valles interandinos y valles de Bolivia $(19,35)$.

Nacobbus aberrans conocido como el nematodo del "rosario de la papa" y Globodera spp. como el "nematodo quiste de la papa", causan tanto daños cuantitativos, por las pérdidas de rendimiento, como cualitativos, por afectar la calidad de los tubérculos-semillas. Estos últimos al ser producidos en campos donde se detecta la incidencia de $N$. aberrans y/o Globodera spp., son descalificados y descienden a la categoría fiscalizada, de acuerdo a normas del Consejo Nacional de Semillas (14).

El efecto negativo en los rendimientos ocasionados por $N$. aberrans y/o Globodera spp., varía con la densidad inicial de estos nemátodos en el suelo, las condiciones climáticas y de suelo, y de la variedad de los cultivos (6).

$N$. aberrans disminuye tanto el rendimiento total de la papa como la cosecha de tubérculos grandes, en respuesta a las densidades de nemátodos en los terrenos infestados $(20,32,39)$, siendo las pérdidas cada vez más elevadas debido al incremento anual de la infestación del campo (20). Además, N. aberrans, a diferencia de Globodera spp., que es un parásito restringido al cultivo de papa y del tomate $(18,22,25)$, posee mayor cantidad de hospedantes que 
comprenden 17 familias, con 69 especies cultivadas y no cultivadas o malezas $(4,24)$.

Los medios de diseminación para ambos nemátodos, son el suelo que va adherido a las herramientas de trabajo, los animales, vehículos, así como también, el agua y el viento. Sin embargo, el principal factor de diseminación de $N$. aberrans es el uso de tubérculos infectados. En el caso de Globodera spp. el principal medio de diseminación es el suelo con quistes adheridos a los tubérculos-semillas.

Una vez iniciada la infestación del suelo por la introducción de cualquiera de los nemátodos es imposible erradicarlos y sólo es posible disminuir su población por medio de un programa de manejo integrado. Esta posibilidad requiere del empleo racional de diversos componentes que no siempre garantizan su efectividad debido a la compleja interrelación de los recursos medioambientales.

Es importante indicar qué la adopción a corto plazo de estos medios combativos contra los nemátodos, significan gastos adicionales que normalmente, no son compensados con la venta de los tubérculos cosechados.

Además, $N$. aberrans se multiplica en diverso grado en cultivos comerciales como la papalisa (Ullucus tuberosus), la oca (Oxalis tuberosa) (13) y la quinua (Chenopodium quinoa) (3); así como también en otras plantas y malezas $(11,12)$. Todos estos hospedantes de $N$. aberrans coadyuvan a que este nematodo incremente su población a través de su infestación a tubérculos como papa, papalisa y algunas ocas e infeste nuevas áreas de producción.

Dentro de esta situación compleja, el conocimiento de la distribución geográfica de $N$. aberrans y Globodera spp. en Bolivia, orienta a las instituciones del quehacer agronómico y las que trabajan con el cultivo de papa, sobre las medidas preventivas que en el futuro se deberán adoptar.

Aun cuando el conocimiento sobre la presencia o ausencia de los dos nemátodos en un área determinada (incidencia), es de gran valor, el conocer el grado de infestación de cada uno de ellos es aún más importante, ya que su relación es directa con las pérdidas de rendimientos (severidad). 
La incidencia de nemátodos en Bolivia es conocida en base a la prospección realizada en diferentes oportunidades en los departamentos de Cochabamba, Potosí, La Paz, Tarija, Chuquisaca y Oruro. Todos estos departamentos cuentan con importarles áreas destinadas a la producción de papa con carácter comercial y de subsistencia y ofrecen condiciones más favorables al desarrollo de los géneros de nemátodos Nacobbus aberrans y Globodera spp. En relación a Santa Cruz, Beni y Pando. Estos últimos tres departamentos, que tienen clima cálido y más favorable a otros géneros de nemátodos, recientemente están incrementando sus áreas de cultivo para la producción de papa consumo.

Es así que, toda la información disponible a nivel nacional, que describe en una u otra forma el uso de estos dos parámetros, ha sido analizada en el presente estudio para estimar las pérdidas en el valor bruto de la producción que tanto $N$. aberrans como Globodera spp. causan en el cultivo de la papa en el país.

\section{Materiales y Métodos}

Diversas fuentes de información constituidas por trabajos publicados y por publicar sobre incidencia y severidad del daño de los nemátodos $N$. aberrans y Globodera spp. en los departamentos de Cochabamba, Potosí, Chuquisaca, Tarija, La Paz y Oruro, fueron recolectadas y revisadas. Estas consistieron en tesis de grado, diagnósticos o informes anuales de diversas instituciones nacionales involucradas en el cultivo de la papa. No se obtuvo información sobre la distribución de estos nemátodos en los departamentos de Santa Cruz, Beni y Pando,

La información recogida fue ordenada y se utilizó para elaborar mapas departamentales. Las comunidades en cuestión fueron ubicadas por provincia de acuerdo al género de nematodo para identificar su ausencia o presencia.

Las diversas fuentes analizadas indican; que la incidencia de $N$. aberrans y Globodera spp. se determinó por medio de prospecciones realizadas en diferentes números de parcelas por comunidad y se la estimó por métodos diferentes y/o coincidentes. Es así que algunos autores $(1,2,8,10,16,17,27,28,33,34,36,37,41)$ calcularon el porcentaje de incidencia de $N$. aberrans y Globodera spp., a través de una fórmula que consideraba el número de parcelas afectadas sobre el número total de parcelas maestreadas. Por otro lado, algunos de los investigadores utilizaron escalas que indicaban la reacción de la planta a la infección de $N$. aberrans. Cada grado de reacción asignado se realizó en base al número de nódulos presentes en las raíces de las plantas extraídas $(5,30)$. Otros investigadores $(5,21)$, 
proporcionaron una información puntual sobre la incidencia de uno o de ambos nemátodos.

En cuanto a la determinación de la severidad de $N$. aberrans y Globodera spp., no todos los documentos consultados proporcionaron esta determinación que es un índice muy relacionado con las pérdidas de rendimientos en el cultivo de papa.

En varios documentos el grado de infestación del suelo por $N$. aberrans, se relacionó al grado de infección radicular, asignado en base al porcentaje de nodulación o el número de nódulos presentes en las raíces extraídas directamente del campo $(2,7,9,10,23,29$, $36,37)$ y de bioensayos $(1,27,40)$.

Las escalas para calificar el grado de infestación de los suelos en algunos casos difirieron (7) y en otros fueron similares $(2,9,10,23$, $29,36,37)$. Sin embargo, algunos autores $(36,37)$ estimaron la severidad de $N$. aberrans por comunidad, utilizando información sobre el grado de ataque en cada una de las parcelas evaluadas con la siguiente fórmula:

$$
\begin{aligned}
& \text { \% Severidad }=\frac{X 1 \times 1+\ldots \ldots \ldots . . \times 4 \times 4}{\mathrm{~A} \times 4} \times 100 \\
& X 1=\text { Número de parcelas con grado } 1 \\
& X 4=\text { Número de parcelas con grado } 4 \\
& \text { A = Número total de parcelas consideradas }
\end{aligned}
$$

Finalmente, Lanza (1996) y Alconz (1997), determinaron el nivel de infestación de los suelos de acuerdo a una escala de cinco grados $(0$, $1,2,3,4)$ que considera el número de individuos (estados infectivos) de $N$. aberrans en $100 \mathrm{~g}$ de suelo.

Considerando esta diversidad de criterios entre autores para definir el grado de infestación de los suelos de acuerdo a la severidad de daño medida por la presencia de nódulos en las raíces y las pérdidas de rendimiento que ocasionan, se estableció para el caso de $N$. aberrans la escala que aparece en la Tabla 1 
Tabla 1. Pérdidas de rendimiento y determinación de los niveles de infestación de los suelos a través del número de nódulos por planta ocasionada por N. aberrans.

\begin{tabular}{lccc}
\hline $\begin{array}{c}\text { Grado } \\
\text { infestación } \\
\text { suelo }\end{array}$ & $\begin{array}{c}\text { No. Indiv./100g } \\
\text { suelo* }\end{array}$ & $\begin{array}{c}\text { No. nódulos/ } \\
\text { planta * }\end{array}$ & $\begin{array}{c}\text { Pérdidas de } \\
\text { rendimiento } \\
\mathbf{( \% ) \star \star ~}\end{array}$ \\
\hline Libre & 0 & 0 & 0 \\
Incipiente & $1-15$ & $1-15$ & 33 \\
Media & $16-30$ & $16-30$ & 68 \\
Alta & $31-70$ & $31-75$ & 77 \\
Muy Alta & $>70$ & $>75$ & 88 \\
\hline
\end{tabular}

* Fuente: Lanza, 1996; Alconz, 1997; Tola, 1997.

** Fuente: Siles, 1996.

La severidad de Globodera spp., en la mayoría de los casos, se estableció por el grado de infestación de los suelos a través del número de quistes por $100 \mathrm{~g}$ de suelo (quistes/100g suelo) $(1,5,17$, $28,33,34)$. Por otra parte, en ciertos casos se determinó la severidad de Globodera spp. a través del número de quistes en raíces directamente extraídas del campo (8) y de acuerdo a una escala que se refería a los niveles o grados de infestación del suelo, mas no a las pérdidas de rendimiento que éstos ocasionaban.

Sin embargo, aún cuando se observó mayor uniformidad de criterios en relación con $N$. aberrans, la correcta determinación de la infestación del suelo por Globodera spp. se debe expresar a través del número de huevos y/o estados juveniles por gramo de suelo (15, 27, 28, 33, 34, 40). Esta determinación evita sobrestimar la infestación del suelo, porque descarta quistes vacíos y huevos no viables. Este criterio, aunque fue muy poco utilizado, se consideró para estimar las pérdidas de rendimiento (Tabla 2), aún cuando tiene el inconveniente que requiere de cierto equipo y de personal entrenado. 
Tabla 2. Pérdidas de rendimiento y niveles de infestación de los suelos por Globodera spp., en base al número de huevos y $\mathrm{J} 2$ por gramo de suelo.

\begin{tabular}{lcc}
\hline $\begin{array}{c}\text { Grado infestación } \\
\text { suelo }\end{array}$ & $\begin{array}{c}\text { Huevos + J2/ gramo } \\
\text { suelo }\end{array}$ & $\begin{array}{c}\text { Pérdidas de } \\
\text { rendimiento (\%) }\end{array}$ \\
\hline Libre & 0 & 0 \\
Incipiente & $1-5$ & 5 \\
Media & $5.1-15$ & 13 \\
Alta & $15.1-35$ & 45 \\
Muy Alta & $>35$ & 58 \\
\hline
\end{tabular}

Fuente: Lanza, 1996; Tola, 1997; Peralta, 1995; Maín, 1994; Esprella, 1993.

La información obtenida sobre la incidencia y los diferentes grados de severidad de $N$. aberrans y Globodera spp. en las áreas prospectadas de los diversos departamentos, más la referente a las áreas afectadas, fue analizada para establecer la situación nematológica actual de los suelos destinados a la producción de papa en Bolivia (26).

La proyección de las pérdidas de rendimiento en relación al grado de severidad de daño por $N$. aberrans y Globodera spp., se estimaron partiendo de los rendimientos promedio por departamento (Tabla 3 ) (42) y de los porcentajes de pérdidas establecidos (Tablas 1 y 2, respectivamente), para cada grado o nivel de infestación del suelo y cuyas denominaciones han sido homologadas para evitar el empleo de diferentes terminologías.

Tabla 3. Rendimientos promedio departamentales a nivel agricultor y áreas cultivadas de papa en Bolivia

\begin{tabular}{|lcc|}
\hline Departamento & $\begin{array}{c}\text { Rendimiento promedio } \\
(\mathrm{kg} / \mathrm{ha})^{\star}\end{array}$ & Área cultivada (ha) \\
\hline Cochabamba & 6759 & 25850 \\
Potosí & 5051 & 30750 \\
La Paz & 5076 & 37800 \\
Tarija & 6942 & 7500 \\
Chuquisaca & 5752 & 19780 \\
Oruro & 3787 & 9650 \\
\hline
\end{tabular}

Fuente: Zeballos, 1997*; INE, 1994** 
Asimismo, esta información junto con los datos de venta y las hectáreas afectadas por grado de severidad fueron utilizadas para estimar las pérdidas económicas que estarían ocasionando $N$. aberrans y Globodera spp. a nivel departamental y nacional.

\section{Resultados y discusión}

A nivel nacional $N$. aberrans, ya sea en forma aislada o asociada con Globodera spp., es el nematodo de mayor cobertura geográfica, ya que se encuentra afectando la producción de papa de la mayoría de las comunidades prospectadas en los departamentos de Cochabamba, Potosí, Tarija, Chuquisaca y Oruro. Al contrario, Globodera spp. aislada o asociada con $N$. aberrans, está afectando más al cultivo de papa en La Paz (Figura 1).

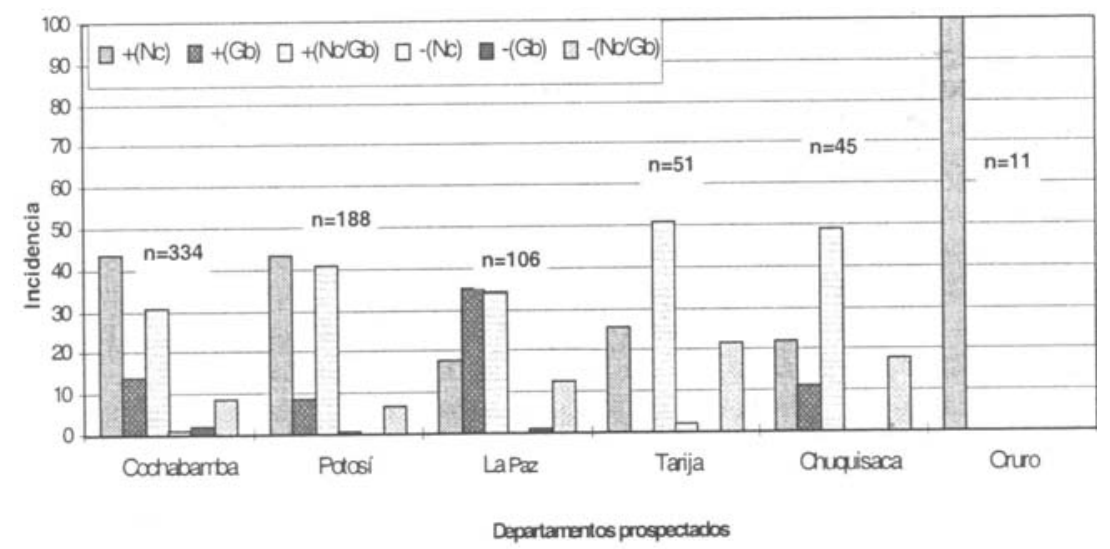

Figura 1. Porcentaje de incidencia de Nacobbus aberrans y Globodera spp. en base al número de comunidades prospectadas (n), en seis departamentos de Bolivia (con (+Nc) o $\sin (-\mathrm{Nc}) \mathrm{N}$. aberrans; con (+Gb) o sin (-Gb) Globodera spp.; con (+Nc/Gb) o $\sin (-\mathrm{Nc} / \mathrm{Gb})$ N. aberrans y Globodera spp.).

En forma absoluta, el número de comunidades con mayor cantidad de parcelas libres de nemátodos se encuentran más en los departamentos de Tarija y Chuquisaca y después en La Paz, Cochabamba y Potosí. Sin embargo, de los cinco departamentos las comunidades con parcelas libres de nemátodos llegan tan sólo a 74 y entre ellas el 46\% fueron prospectadas por Caero en 1984. 
Actualmente, es muy difícil confiar que las mismas comunidades cuenten con similar probabilidad de presentar parcelas libres de nemátodos. La adquisición de semilla de papa de forma informal por parte del agricultor juega un papel decisivo en esta suposición.

Los porcentajes de incidencia establecidos han sido utilizados para estimar el número de hectáreas afectadas por $N$. aberrans y/o Globodera spp. en base al número de hectáreas destinadas al cultivo de papa por departamento (26), y, por lo tanto, reflejan las mismas tendencias señaladas para los porcentajes de incidencia por departamentos y cuya información se utilizará posteriormente para estimar las pérdidas económicas. Estas pérdidas son presentadas en forma de valor bruto de la producción total del país afectada, de acuerdo a la severidad de daño ocasionado por ambos nemátodos. Por otro lado, la prospección de las comunidades para conocer la severidad de $N$. aberrans en los diferentes departamentos fue mayor que la realizada para Globodera spp. (287 versus 229 comunidades). Sin embargo, ambas cantidades, aún sumadas, no llegan ni a igualar al número de comunidades prospectadas para conocer la incidencia. Se llegó a determinar que las infestaciones de los suelos por $N$. aberrans en Bolivia son mayores que aquellas producidas por Globodera spp.

$N$. aberrans se encuentra infestando los suelos de los departamentos prospectados mayormente en gradaciones incipientes $(49.36 \%$ de las comunidades) y medias ( $22.46 \%$ de las comunidades). Solamente en Tarija se llegó a notar cierto cambio en esta tendencia, ya que la proporción de las comunidades afectadas con gradaciones altas llegan a igualar a la proporción de comunidades afectadas con gradaciones medias (Figura 2). Hay que tener en consideración que en la actualidad las infestaciones medias ya están tomando importancia en el ámbito nacional y más claramente en Cochabamba.

Globodera spp. se encuentra infestando los suelos mayormente con poblaciones incipientes ( $29.5 \%$ de las comunidades) y medias $(25.9 \%$ de las comunidades). Sin embargo, las parcelas libres de este nemátodo también tienen cierta importancia en el $21.58 \%$ de las comunidades (Figura 3). 


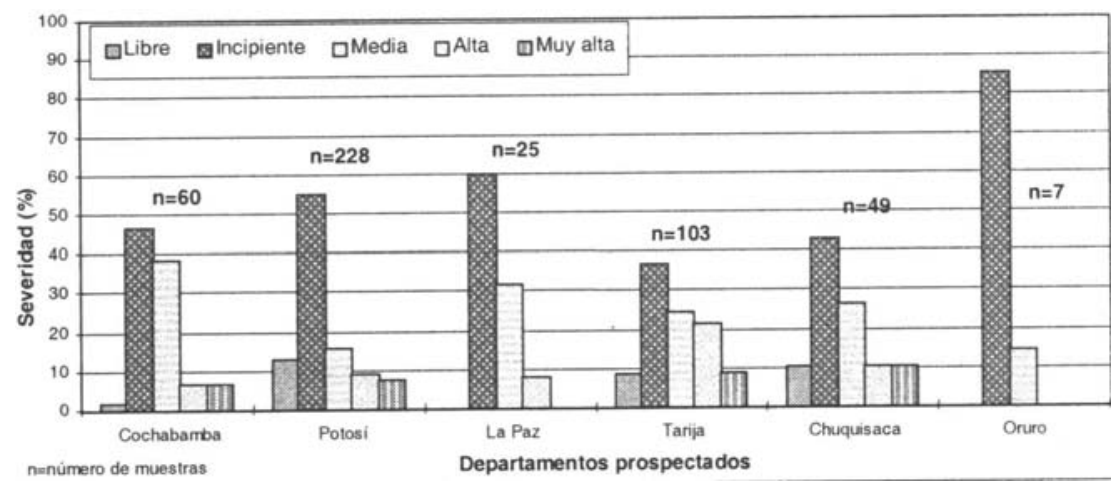

Figura 2. Porcentaje de severidad, por efecto de la infestación de los suelos por Nacobbus aberrans en seis departamentos prospectados en Bolivia.

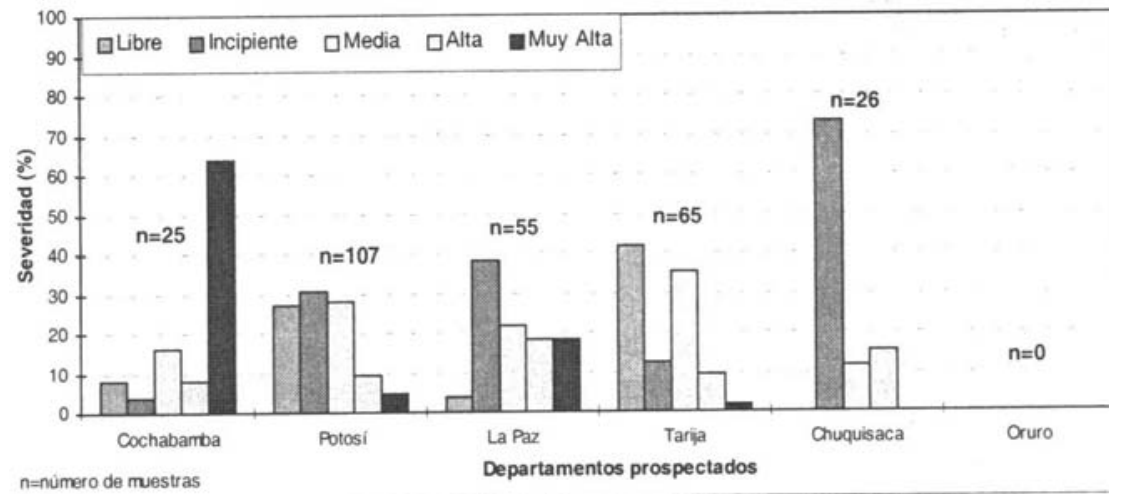

Figura 3. Porcentaje de severidad por efecto de la infestación de los suelos por Globodera spp. en seis departamentos prospectados en Bolivia.

Por el momento las infestaciones por Globodera spp. ganan mayor interés en los departamentos de Cochabamba y La Paz; en este último departamento, las gradaciones altas y muy altas de Globodera spp. están ocupando similar importancia con las infestaciones medias en las diferentes comunidades prospectadas.

Estos resultados son alarmantes, especialmente porque las pérdidas en el rendimiento del cultivo de papa en Bolivia ocasionadas por 
N. aberrans son más marcadas en relación a las ocasionadas por Globodera spp., aún cuando las poblaciones de N. aberrans en los suelos sean incipientes (Figura 4 y Figura 5).

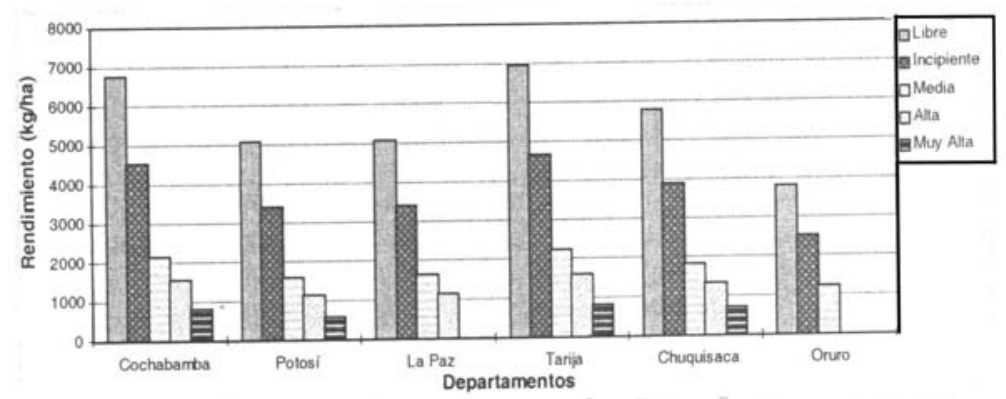

Figura 4. Pérdidas en el rendimiento del cultivo de papa ocasionadas de acuerdo al grado de severidad de daño causado por Nacobbus aberrans en los diferentes departamentos prospectados en Bolivia.

La disminución de los rendimientos por efecto de la infección de cualesquiera de los dos géneros de nemátodos siguen la misma tendencia que el porcentaje de pérdidas ya establecidos para $N$. aberrans (38) y Globodera spp. (27), y en base a los cuales se han realizado los cálculos de pérdidas de rendimientos.

N. aberrans, aún en poblaciones incipientes, afecta significativamente los rendimientos y más aún cuando la infestación es igual o mayor al grado medio. Con Globodera spp. las pérdidas de rendimiento siguen un comportamiento diferente; cuando las poblaciones en el suelo son incipientes y medias, los rendimientos no sufren pérdidas significativas, pero cuando las poblaciones son mayores a las de gradación media, los rendimientos se reducen significativamente. 


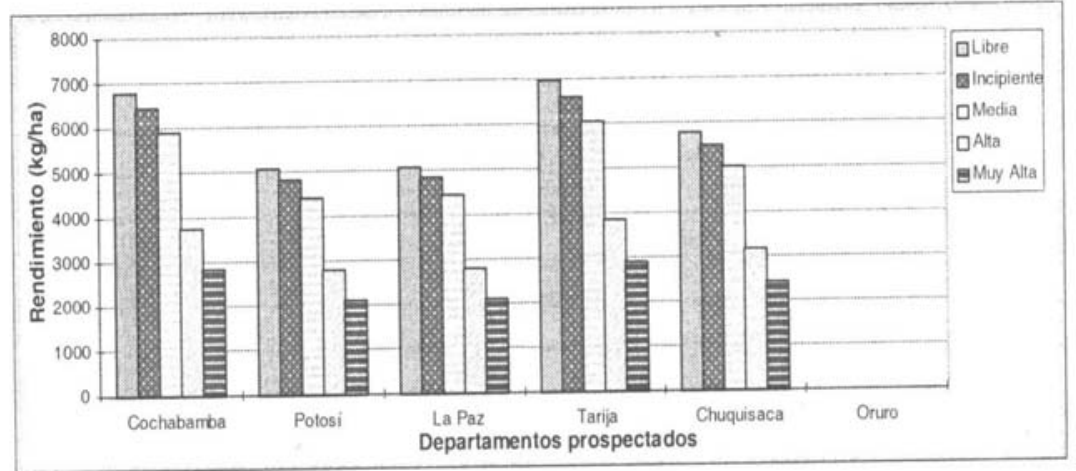

Figura 5. Pérdidas en el rendimiento del cultivo de papa ocasionados de acuerdo al grado de severidad de daño causado por Globodera spp. en los diferentes departamentos prospectados en Bolivia.

Esto conduce a comprender la importancia que tiene el no permitir que los suelos limpios sean infestados especialmente por N. aberrans, porque, a corto plazo, este nematodo en comparación con Globodera spp. incide significativamente en los rendimientos. También estos resultados permiten comprender la importancia de evitar que las infestaciones por $N$. aberrans lleguen a niveles mayores que los incipientes, y a niveles mayores que los medios en el caso de Globodera spp., cuidando que por este medio no se pierda a través de los años la capacidad productiva de los suelos.

En la estimación del número de hectáreas afectadas por grado de severidad de N. aberrans y Globodera spp., primero se calcularon las hectáreas que corresponderían a cada uno de los seis grupos formados de acuerdo a su ausencia o presencia $(+\mathrm{Nc},+\mathrm{Gb},+\mathrm{Nc} / \mathrm{Gb},-$ $\mathrm{Nc},-\mathrm{Gb},-\mathrm{Nc} / \mathrm{Gb}$ ), utilizando el dato del número total de hectáreas destinadas al cultivo de papa por departamento (26) y sus correspondientes porcentajes de incidencia calculados en estos seis grupos.

Posteriormente, la superficie calculada en el grupo $+\mathrm{Nc} / \mathrm{Gb}$ se dividió por la mitad. Cada mitad de superficie del grupo $+\mathrm{Nc} / \mathrm{Gb}$ se sumó por separado con la superficie individualmente afectada por $N$. aberrans y la otra mitad con la superficie infestada solamente por Globodera spp. En cada uno de los dos nuevos grupos (+Nc) y $(+\mathrm{Gb})$ así conformados, se calcularon las hectáreas que estarían siendo afectadas por grado de severidad (libre, incipiente, media, alta y muy 
alta), utilizando los porcentajes de incidencia calculados en base al número de muestras (parcelas) registradas por grado de severidad.

Finalmente, las hectáreas estimadas como libres de $N$. aberrans o de Globodera spp. dentro de las gradaciones de severidad para cada especie, en forma correspondiente llegaron a ser sumadas junto con las hectáreas que fueron estimadas de acuerdo al número de comunidades consideradas como libres de $N$. aberrans o libres de Globodera spp.

De acuerdo a estos cálculos, se observó que el grado incipiente de $N$. aberrans afecta más hectáreas en todos los departamentos prospectados (55.72\%), llegando a ocupar un segundo lugar aquellas afectadas con grado medio (25.33\%). Las hectáreas afectadas con grado incipiente fueron más numerosas que las con gradación media en los departamentos de Potosí, Oruro y La Paz; en cambio, las hectáreas con grado medio son más numerosas, respecto a las de grado incipiente, en los departamentos de Cochabamba, La Paz y Potosí (Figura 6).

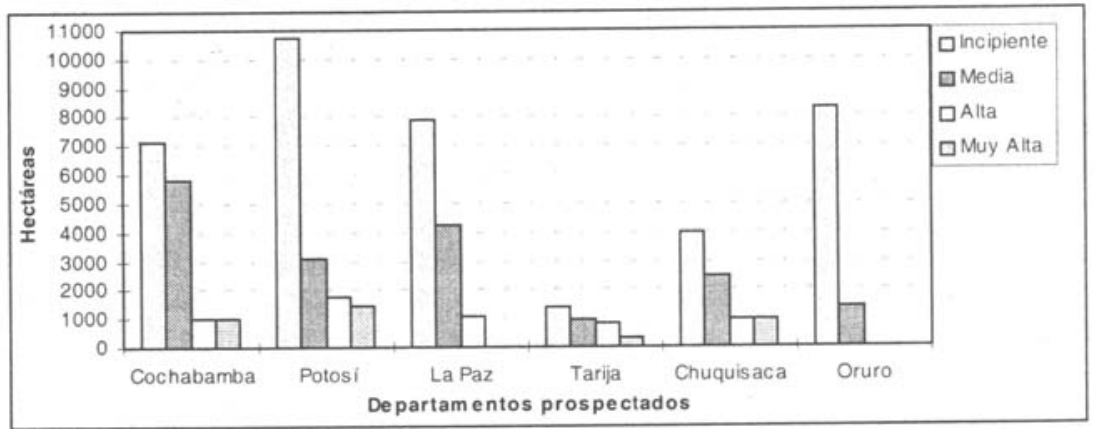

Figura 6. Superficies afectadas (ha) de acuerdo al grado de severidad de daño causado por N. aberrans en los departamentos prospectados.

En el caso de Globodera spp., también las infestaciones incipientes ganan mayor importancia en relación de los demás grados de infestación (35.33\%), afectando mayor número de hectáreas en La Paz, Chuquisaca y Potosí. Seguidamente, las infestaciones medias $(21.04 \%)$ y muy altas (19.18\%) tienen similar importancia. Las hectáreas afectadas por poblaciones medias, se observan más en los departamentos de La Paz y Potosí y las afectadas por poblaciones muy altas en Cochabamba y La Paz (Figura 7). 


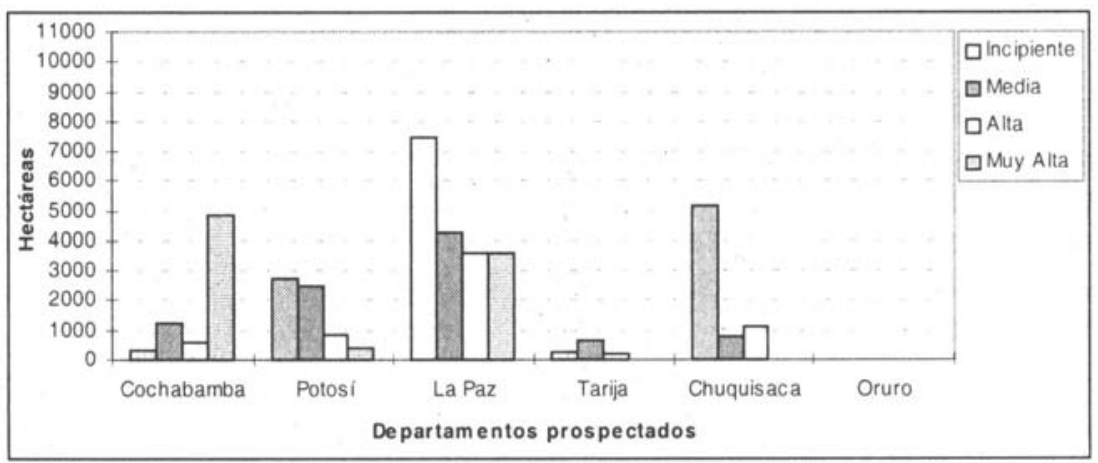

Figura 7. Superficies afectadas (ha) de acuerdo al grado de severidad de daño causado por Globodera spp. en los departamentos prospectados.

Considerando esta información con respecto a $N$. aberrans, a corto plazo debe preocupar las hectáreas afectadas con poblaciones incipientes (constituyen la mayoría), éstas se deben manejar de tal manera que las poblaciones de nemátodos se reduzcan o en todo caso se mantengan. Para ello no permitir que las poblaciones se incrementen, ya que las pérdidas son mayores desde que las poblaciones de nemátodos alcanzan el grado medio.

Las hectáreas afectadas por $N$. aberrans con grado medio, menores en número a aquellas afectadas con grado incipiente en más del $50 \%$, llegan a aumentar significativamente las pérdidas $(38.23 \%$ : US $\$ 19795,051$ ) que es ligeramente mayor a las pérdidas que ocasionan las hectáreas infestadas con grado incipiente (37.42\%: US\$19'375,281).

Una situación muy diferente se observa con el género Globodera spp. Las hectáreas afectadas por infestaciones muy altas son las que están haciendo incurrir en fuertes pérdidas económicas, principalmente en los departamentos de Cochabamba y La Paz V Seguidamente las hectáreas afectadas por poblaciones altas en La Paz y Chuquisaca.

La información recabada hasta este punto, concernientes a las pérdidas de rendimiento en base a los rendimientos promedio departamentales (42) y las hectáreas afectadas por grado de severidad de $N$. aberrans y Globodera spp., combinados con el precio de venta al consumidor por kilogramo de papa (1.5 bs/ kg papa), arrojaron como resultado que las pérdidas (US\$/ha) en el valor bruto de la producción de papa son siempre mayores por efecto de la 
infestación por $N$. aberrans en comparación de las pérdidas causadas por Globodera spp. (Figura 8 y Figura 9).

Con N. aberrans, desde que las infestaciones son de gradación media, las pérdidas económicas son considerables y varían de 733 US\$/ha (infestación media en Oruro) a 1,739 US\$/ha (infestación muy alta en Tarija). Para con Globodera spp. desde que las infestaciones son de gradación alta las pérdidas económicas varían de 647 US\$/ha (infestación alta en Potosí) a 1,146 US\$ /ha (infestación muy alta en Tarija).

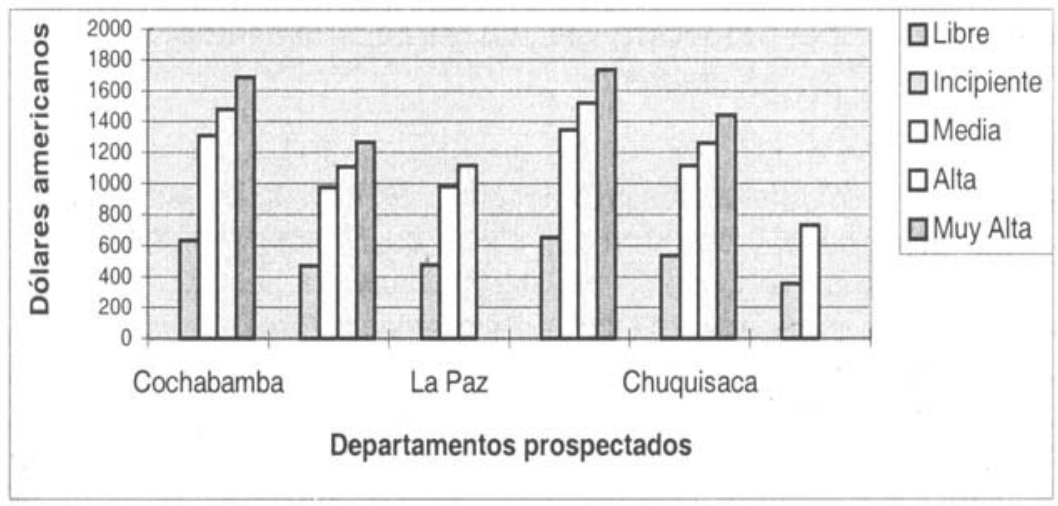

Figura 8. Pérdidas estimadas en el valor bruto de la producción de papa (US\$/ha) de acuerdo a la severidad de daño por $\mathrm{N}$. aberrans, en los departamentos prospectados.

Generalizando estas últimas estimaciones y considerando el total de hectáreas afectadas por grado de infestación de los dos géneros de nemátodos (Figura 10), se desprende que las pérdidas económicas en el valor bruto de la producción de papa en Bolivia son mayores por efecto de las infestaciones por $N$. aberrans (52 millones de dólares aproximadamente), en comparación de aquellas producidas por Globodera spp. (16 millones de dólares aproximadamente). 


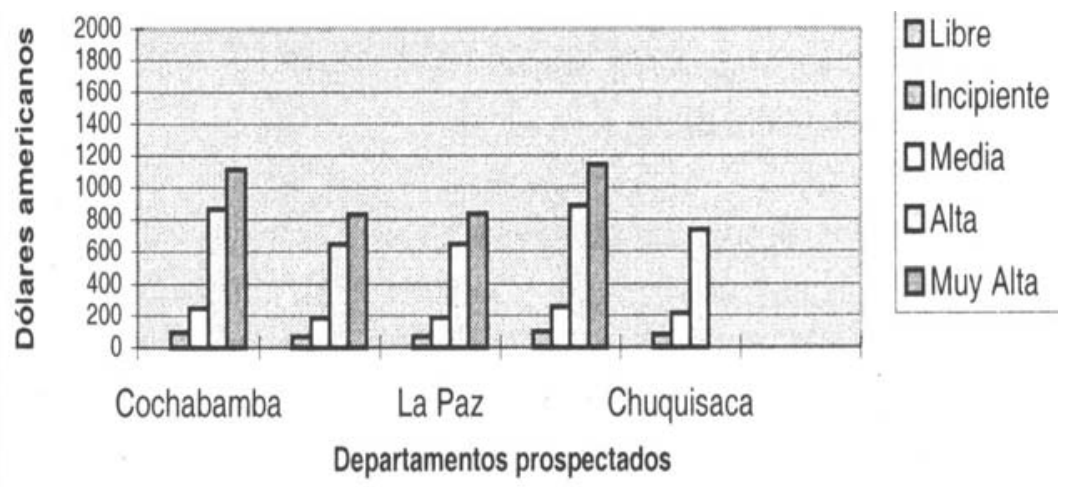

Figura 9. Pérdidas estimadas en el valor bruto de la producción de papa (US\$/ha) de acuerdo a la severidad de daño por Globodera spp., en los departamentos prospectados.

En los seis departamentos prospectados, del total de las pérdidas en el valor bruto de la producción por efecto de $N$. aberrans, los mayores porcentajes corresponden a las infestaciones por poblaciones medias (38.23\%) e incipientes (37.42\%). Asimismo, del total de las pérdidas por Globodera spp., el $54.06 \%$ corresponden a las infestaciones por poblaciones muy altas y el $26.68 \%$ a las infestaciones por poblaciones altas.

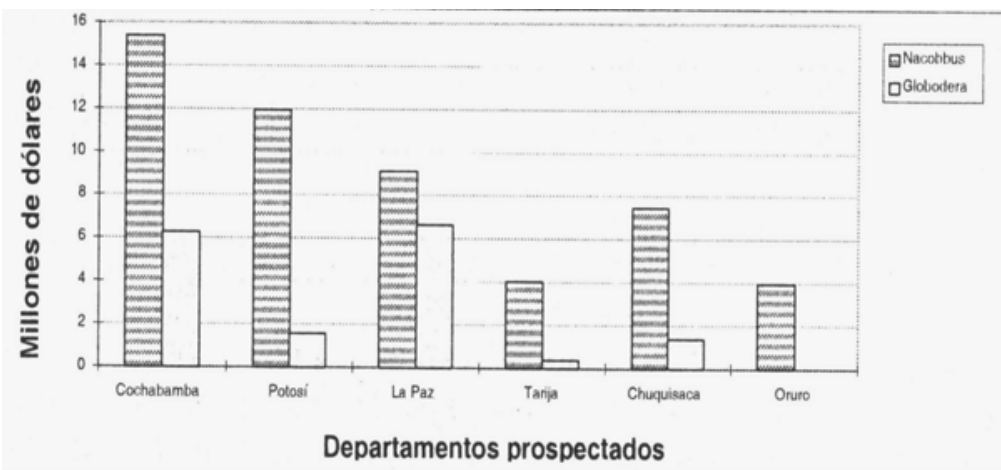

Figura 10. Pérdidas estimadas en el valor bruto de la producción (US\$) causadas por Nacobbus aberrans y Globodera spp. en el cultivo de papa en Bolivia.

Por otro lado, y considerando que la presencia de nemátodos ocasiona pérdidas indirectas en parcelas semilleras de Cochabamba, se ha determinado que en los últimos tres años de una muestra de 279 a 328 hectáreas supuestamente libres de nemátodos para la producción de semilla de papa, se han estado descartando terrenos en 30 a $25 \%$ por constatarse en ellos la presencia de $N$. aberrans. Con 
Globodera spp. el descarte de terrenos por la presencia de éste nematodo que alcanza de 1.8 a $2.4 \%$ es mucho más bajo de lo que muestran las estimaciones (Figura 11).

Por otra parte, se debe hacer notar que a partir de la campaña 1995-96, la incursión de Meloidogyne spp. en las zonas de altura empieza a reducir en $0.18 \%$ las hectáreas para la producción de semillas.

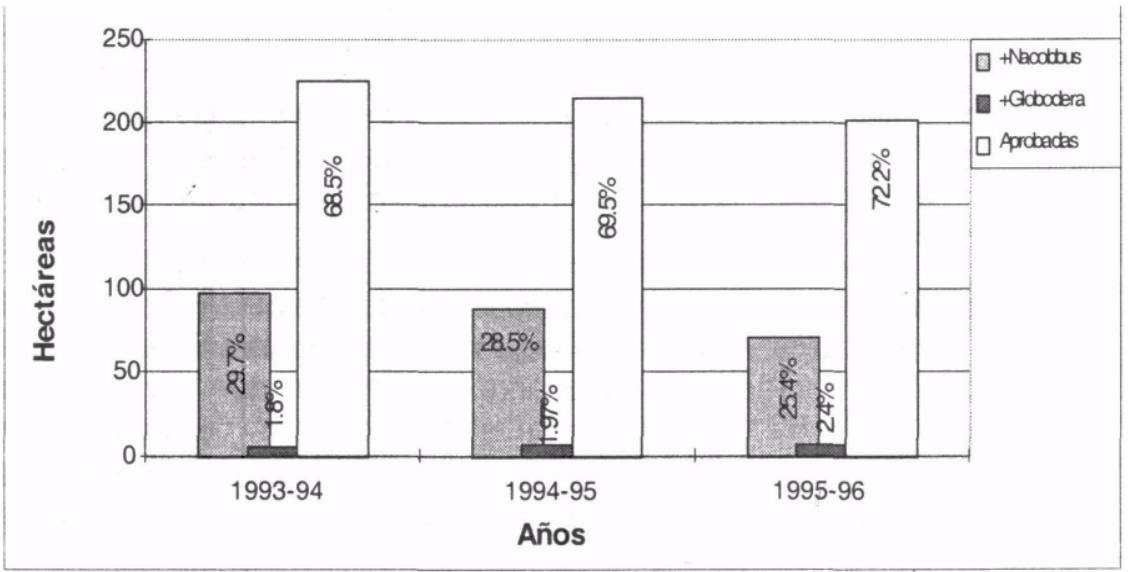

Figura 11. Hectáreas aprobadas y rechazadas por la presencia de N. aberrans y Globodera spp. durante tres campañas agrícolas en Cochabamba.

Sin embargo, especialmente con $N$. aberrans, el porcentaje de parcelas descalificadas para la producción de semilla de papa ha disminuido ligeramente (Figura 11), como consecuencia del desarrollo de tecnologías en el diagnóstico de nemátodos, tal como los bioensayos en bolsas cerradas (31).

Para la campaña 1995-96, en Cochabamba las hectáreas rechazadas para la producción de semillas llegan al $27.98 \%$ y en Potosí al 32.46\%. Ambos son dos de los departamentos con mayor número de hectáreas inspeccionadas y rechazadas, a diferencia de Tarija y Chuquisaca, en las que se encuentran más hectáreas libres de nemátodos en relación del número de hectáreas inspeccionadas (Figura12). 


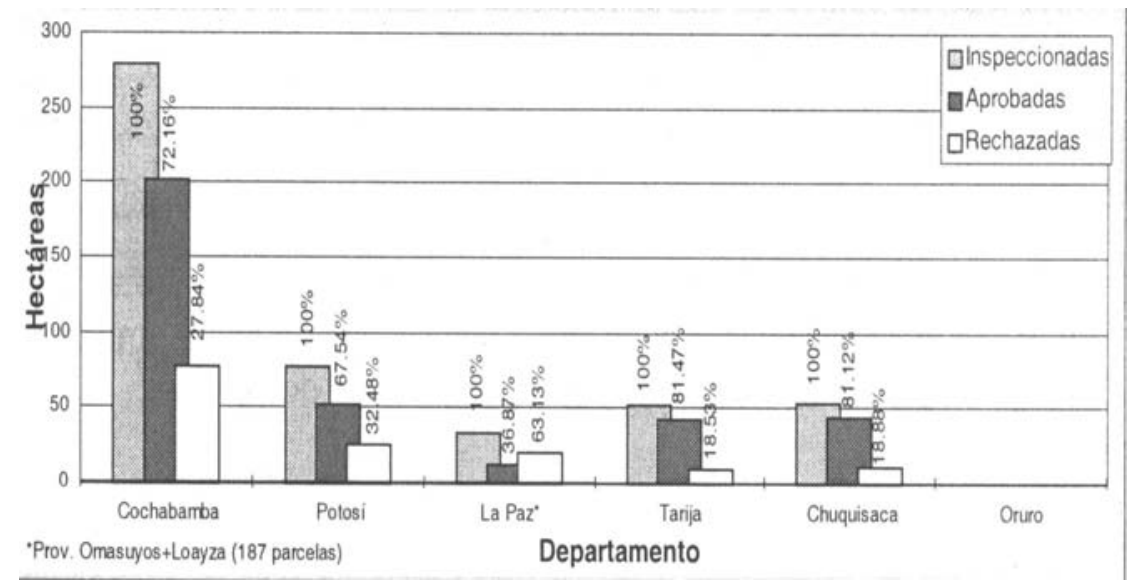

Figura 12. Hectáreas aprobadas y rechazadas en la producción de tubérculos semilla de papa en la Campaña agrícola 1995-96, según los Consejos Regionales de Semillas.

El número de hectáreas inspeccionadas en La Paz es aún extraoficial porque sólo abarca 187 parcelas en las provincias Omasuyos y Loayza. Sin embargo, es importante mencionar que en las dos provincias de 32.57 ha, Globodera spp. está más distribuido que $N$. aberrans, contribuyendo el primero en un $40 \%$ y el segundo en un $23 \%$, en el descarte de nuevas parcelas para la producción de semillas.

\section{Determinación de especies de Globodera spp. en Bolivia}

Los estudios sobre la distribución geográfica de Globodera spp. según la especie se realizó en los departamentos de La Paz, Tarija, Chuquisaca y Cochabamba. No se realizó esta determinación de especies en Potosí y Oruro.

El número de comunidades prospectadas para determinar la especie de Globodera spp. en La Paz y Tarija fue mayor, pero en Cochabamba esta determinación fue más fidedigna por considerarse mayor tamaño de muestra (100 parcelas por comunidad).

En el ámbito nacional, de 815 parcelas con presencia de quistes (54.84\% de 1,855 parcelas totales muestreadas), Globodera pallida superó en $9.9 \%$ la incidencia de Globodera rostochiensis (Figura 13). Chuquisaca fue el más afectado por esta especie y luego La Paz y Cochabamba. En cambio en Tarija y nuevamente en La Paz, la 
presencia de Globodera rostochiensis es más frecuente. Por otro lado, en Tarija se realizó una determinación distinta a los demás departamentos, donde, se observaron $65(17.2 \%)$ parcelas con la presencia simultánea de $G$. pallida y $G$. rostochiensis.

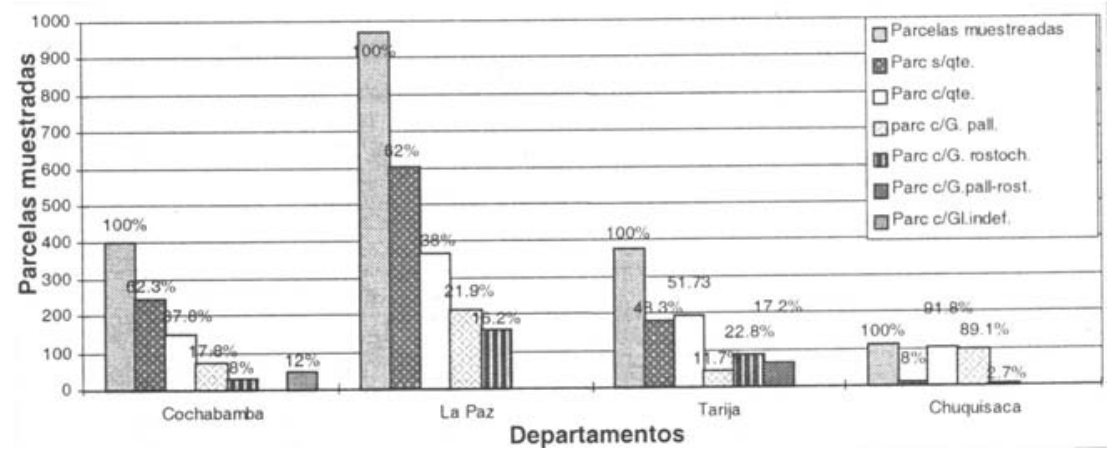

Figura. 13. Incidencia de Globodera pallida y Globodera rostochiensis de acuerdo al número de parcelas muestreadas por departamento.

\section{Distribución de Nacobbus aberrans y Globodera spp. en diferentes pisos altitudinales en Bolivia}

La distribución de nemátodos de acuerdo a la altitud en Bolivia, se realizó en base a 503 comunidades con altitud conocida (Figura 14).

Entre los 3,001 a 3,500 msnm y los 3,501 a 4,000 se encuentran concentrados el mayor número de comunidades afectadas por nemátodos (35.39 y 31.41\%), principalmente por Nacobbus aberrans en forma individual, como simultáneamente con Globodera spp. La presencia de Globodera spp. en forma individual fue más notable en comunidades entre los 3,501 y 4,000 msnm.

En casi todas las altitudes consideradas en este estudio, la presencia simultánea de $N$. aberrans y Globodera spp. y la individual de $N$. aberrans, fueron las formas más comunes.

Por otra parte, por debajo de los 3,000 msnm se observó mayor número de comunidades con parcelas libres de nemátodos (-Na/Gb) y se explica por la baja adaptación de $N$. aberrans y Globodera spp. a temperaturas promedio altas $\left(>20^{\circ} \mathrm{C}\right)$, que a su vez, están inversamente asociadas con la altitud. Esta tendencia es más relevante debajo de los 2,000 msnm, tal como se observó en el 
departamento de Tarija, donde los porcentajes de incidencia de las comunidades libres de nemátodos son mayores, en relación al total de comunidades prospectadas por rango de altitud.

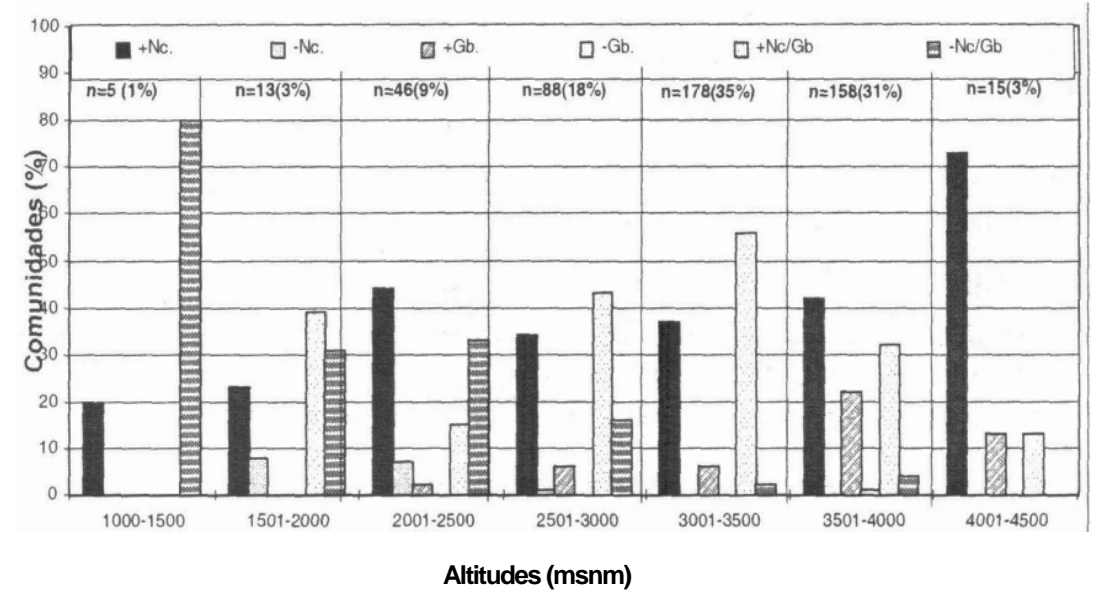

Figura 14. Incidencia de Nacobbus aberrans y Globodera spp. de acuerdo al número de comunidades en los diferentes pisos altitudinales.

\section{Conclusiones}

Los resultados y análisis sobre la incidencia y severidad de $N$. aberrans y Globodera spp., y distribución de especies de Globodera spp. a nivel nacional, indican que $N$. aberrans en forma individual como simultáneamente con Globodera spp. se encuentra ampliamente distribuido a nivel nacional, afectando mayores superficies de terrenos. En cambio, la distribución geográfica como la superficie afectada por Globodera spp. está más concentrada en La Paz y Potosí.

La severidad de daño por $N$. aberrans es mucho más marcada que la severidad por Globodera spp., y está afectando la productividad del cultivo de papa en poblaciones incipientes y medias. Solamente en Tarija, las infestaciones altas tienen similar importancia que las infestaciones medias. También Globodera spp. se encuentra más en poblaciones incipientes y medias. Sin embargo, la ausencia de este nematodo, también es notable en relación a las infestaciones por poblaciones altas y muy altas. 
Las poblaciones incipientes de $N$. aberrans tienen mayor trascendencia y abarcan grandes superficies de terrenos en Potosí, Oruro y La Paz. Posteriormente las infestaciones de grado medio abarcan grandes superficies en Cochabamba, La Paz y Potosí.

Las poblaciones incipientes de Globodera spp. tienen mayor trascendencia y abarcan grandes superficies de terrenos en la Paz, Chuquisaca y Potosí. Las infestaciones de grado medio y muy alto, abarcan idénticas superficies, las poblaciones medias se observan más en La Paz y Potosí y las poblaciones muy altas en Cochabamba y La Paz.

N. aberrans desde poblaciones medias y Globodera spp. desde poblaciones altas en los suelos, incrementan significativamente las pérdidas económicas.

En base a rendimientos promedio departamentales, las pérdidas causadas por $N$. aberrans varían de 733 US\$/ha en poblaciones medias a 1,739 US\$/ha en poblaciones muy altas. Si las pérdidas se dan por efecto de Globodera spp. Ias pérdidas aumentan de 647 US\$/ha en poblaciones altas a 1,146 US\$/ha en poblaciones muy altas.

Las pérdidas en el valor bruto de la producción de papa en Bolivia son mayores por efecto de la infestación de $N$. aberrans, llegando a totalizar alrededor de 52 millones de dólares, en comparación de aquellas provocadas por Globodera spp. que llegan a ser superiores a 16 millones de dólares.

Del total de las pérdidas nacionales en el valor bruto de la producción de papa por efecto de $N$. aberrans, el $38.23 \%$ corresponden a las causadas por poblaciones medias y el $37.42 \%$ por poblaciones incipientes. Por otro lado, del total de las pérdidas nacionales por efecto de Globodera spp., el $54.06 \%$ corresponden a las causadas por poblaciones muy altas y el $26.68 \%$ por poblaciones altas.

Potosí, Cochabamba y La Paz son los departamentos que presentan las mayores pérdidas económicas por poblaciones incipientes y medias de $N$. aberrans. Por otro lado, Potosí, Cochabamba y Chuquisaca son los que presentan las mayores pérdidas por poblaciones altas y muy altas.

Con el género Globodera spp., Cochabamba y La Paz presentan las mayores pérdidas en el valor bruto de la producción de papa por 
poblaciones de gradación muy alta. Seguidamente, La Paz, Chuquisaca y Potosí presentan las mayores pérdidas por poblaciones altas. Por último, La Paz, Potosí y Chuquisaca presentan las mayores pérdidas por poblaciones medias e incipientes de Globodera spp.

Por la presencia de nemátodos, Cochabamba y Potosí son los departamentos donde se inspeccionan y se descartan a la vez, un mayor número de hectáreas. En cambio, en Tarija y Chuquisaca, se encuentran más hectáreas libres de nemátodos en relación al número de hectáreas inspeccionadas.

En Bolivia, Globodera pallida supera ligeramente la incidencia de Globodera rostochiensis. Chuquisaca, La Paz y Cochabamba son los más infestados por esta especie, en cambio, Tarija y La Paz por G. rostochiensis. Por otro lado, Tarija también se encuentra simultáneamente infestada por G. pallida y G. rostochiensis.

La diferencia de altitud no altera la incidencia dominante de $N$. aberrans ya sea en forma individual como simultáneamente con Globodera spp., sin embargo, las altitudes entre los 3,000 a 4,000 msnm concentran a un mayor número de comunidades afectadas por estas dos formas de incidencia de $N$. aberrans. La incidencia de Globodera spp. es más notable en comunidades entre los 3.500 a 4,000 msnm.

Las parcelas libres de nemátodos son más frecuentes entre los 1,000 a 3,000 msnm.

La ausencia o menor presencia de $N$. aberrans y Globodera spp. en altitudes menores a los 3,000 msnm, se relaciona con la menor frecuencia del cultivo de papa en comparación al cultivo de otras especies de plantas y con las temperaturas prevalecientes mayores a los $20^{\circ} \mathrm{C}$, desfavorables al desarrollo de estos dos nemátodos.

La ausencia o menor presencia de $N$. aberrans y Globodera spp. en altitudes mayores a los 3,000 msnm, guardan relación con su lejanía de los caminos de fácil acceso vehicular, el uso de semilla propia de papa por parte del agricultor, con el descanso prolongado de los suelos (>5 años) y con las rotaciones de cultivos con leguminosas y cereales. 


\section{Referencias Bibliográficas}

1. Alconz, E. 1997. Mapeo de suelos para nemátodos en la provincia Ayopaya del departamento de Cochabamba. Tesis Ing. Agr., Universidad Mayor de San Andrés. La Paz, Bolivia. 119 p.

2. Ali, R. 1995. Distribución de Nacobbus aberrans y grado de infestación en la zona de Lequezana, Potosí. Tesis Ing. Agr., Universidad Autónoma "Tomás Frías". Potosí, Bolivia. 87 p.

3. Barker, K. 1986. Informe sobre revisión de problemas de nemátodos parásitos de plantas en Bolivia para el Proyecto de Control Integrado de Plagas en papa y tomate. Proyecto de Control Integrado de Plagas PROCIPLA/USAID. $11 \mathrm{p}$.

4. Brodie, B. B.; Evans, K.; Franco, J. 1993. Nematode Parasites of Potato. En. Plant Parasitic Nematodes in Temperate Agriculture. K. Evans, D. Trudgill and J. Webster (eds). CAB International, United Kingdom. p. 87-132.

5. Caero, G. 1984. Distribución de nemátodos de importancia económica en zonas productoras de papa en Bolivia. Tesis Ing. Agr., Universidad Mayor de San Simón. Cochabamba, Bolivia. 126p.

6. Canto-Sáenz, M. 1987. Los nemátodos y la producción de papa. En. El cultivo de papa con énfasis en la producción de semilla. Programa de Investigaciones y Proyección Social en Papa, Universidad Nacional Agraria "La Molina". Lima, Perú. p. 193-212.

7. Casso, R. 1992. Fotocopias de tablas sobre incidencia y/o severidad de $N$. aberrans y/o Globodera spp. en el departamento de Tarija. Tarija, Bolivia. $4 \mathrm{p}$.

8. Casso, R. 1993. Distribución de especies de Globodera spp. en Tarija. En Informe Anual IBTA-PROINPA 1992-93. Cochabamba, Bolivia. p. 318-319.

9. Casso, R.; Franco, J. 1993a. Distribución y hospedantes de Nacobbus aberrans en Tarija. En Informe Anual IBTA-PROINPA 1992-93. Cochabamba, Bolivia. p. 250-251.

10. Casso, R.; Franco, J. 1993b. Comportamiento de cultivares (cv. Gendarme) a Nacobbus aberrans en tres localidades del departamento de Tarija. En: Informe Anual IBTA-PROINPA 1992-93. Cochabamba, Bolivia. p. 252-256.

11. Castiblanco, A. 1992. Razas y gama de hospedantes en diferentes poblaciones de Nacobbus aberrans. Tesis Técnico Superior Agrónomo, Universidad Mayor de San Simón. Cochabamba Bolivia. $73 \mathrm{p}$. 
12. Céspedes, L. 1994. Comportamiento de diferentes especies vegetales a la invasión y desarrollo de Nacobbus aberrans. Tesis Técnico Superior Agrónomo, Universidad Mayor de San Simón. Cochabamba, Bolivia. $60 \mathrm{p}$.

13. Condori, P. ; Franco, J. 1994. Evaluación de cultivos andinos al ataque de Nacobbus aberrans. En: Informe Anual IBTA-PROINPA 1993-94. pp. 50 III.N.-52 III.N.

14. Consejo Nacional de Semillas. 1995. IX Mesa Redonda Nacional sobre semillas - Memoria. Sucre, Bolivia. $67 \mathrm{p}$.

15. Esprella, R. 1993. Evaluación en parcelas campesinas del nematodo del quiste de la papa (Globodera spp.) en función al tiempo de descanso en el Altiplano Central Boliviano. Tesis Ing. Agr., UMSS. Cochabamba, Bolivia. $99 \mathrm{p}$.

16. Fernández, J. 1991. Patógenos de importancia económica y recursos para producción de semilla de papa en zonas paperas de la provincia Carrasco. Tesis Ing. Agr., Universidad Mayor de San Simón. 125p.

17. Flores Ch., L. 1996. Mapeo nematológico (N. aberrans y Globodera spp.) en las principales áreas paperas de la provincia Modesto Omiste, Potosí. Tesis Ing. Agr., Universidad Autónoma 'Tomás Frías". Potosí, Bolivia. 127 p.

18. Franco, J. 1986. Nemátodos del quiste de la papa. Boletín de Información Técnica. 19 p.

19. Franco, J.; Montecinos, R.; Ortuño, N. 1992. Nacobbus aberrans, nematodo fitoparásito del cultivo de la papa en Bolivia: Desarrollo de una estrategia para su Manejo Integrado. IBTA-PROINPA/F.C.A.P.UMSS. Revista de Agricultura 21: 11-22. Cochabamba, Bolivia.

20. Franco, J. 1994. Problemas de nemátodos en la producción de papa en climas templados en la región andina. Nematrópica 24: 179-195.

21. Franco, J.; Maín, G. 1995. Distribución e identificación de especies de Globodera spp. en el departamento de Cochabamba. En: Informe Anual IBTA-PROINPA 1994-95. Cochabamba, Bolivia. p. 73 III.N.-76 III.N.

22. Hooker, J. 1980. Compendio de enfermedades de la papa. Centro Internacional de la Papa (CIP). Lima, Perú.. 486p. 
23. Ibarra, R.; Franco, J.; Montecinos, R. 1992. Comportamiento de un cultivar parcialmente resistente (cv. Gendarme) al ataque de Nacobbus aberrans bajo diversas condiciones agroecológicas (Lequezana, Potosí). En: Informe Anual IBTA-PROINPA 1991-92. Cochabamba, Bolivia, pp. 148-150.

24. IBTA-PROINPA. 1992. Diagnóstico interdisciplinario del cultivo de papa en Cochabamba (Jatum Tarpuy 1989-1990). Documento de trabajo 2/92. Cochabamba, Bolivia. 106p.

25. Instituto Nacional de Investigación y Promoción Agropecuaria (INIPA); Programa Andino Cooperativo de Investigación en Papa (PRACIPA). 1987. Manejo integrado de principales plagas de papa en Puno. Puno, Perú. 14 p.

26. Instituto Nacional de Estadística (INE); Ministerio de Desarrollo Sostenible y Medio Ambiente. 1994. Boletín de Estadísticas Agrícolas 1992-93-1994. La Paz, Bolivia. 53 p.

27. Lanza, M. E. 1996. Distribución de Nacobbus aberrans y Globodera spp. en zonas paperas de siete provincias del departamento de Potosí. Tesis Ing. Agr., Universidad Autónoma 'Tomás Frías". Potosí, Bolivia. 75 p.

28. Maín, G. 1994. Distribución de Globodera spp. en cuatro provincias de La Paz e identificación de fuentes de resistencia. Tesis Ing. Agr., Universidad Técnica de Oruro. Oruro, Bolivia. 94 P.

29. Montecinos C., W. 1991. Influencia del Ph de suelo en la densidad poblacional de Nacobbus aberrans (Thorne, 1935), Thorne y Allen 1944, en el cultivo de papa. Tesis Ing. Agr., Universidad Mayor de San Simón. Cochabamba, Bolivia. 120 p.

30. O Navia, R. 1989. Efecto residual del cultivo de piretro Chrysantemun cinerariaefolium sobre poblaciones de Nacobbus aberrans en tres variedades de papa. Tesis Ing. Agr., Universidad Mayor de San Simón. Cochabamba, Bolivia. 89 p.

31. Ortuño, N.; Oros, R.; Maín, G.; Franco, J. 1996. Detección de nemátodos por el método de la bolsa cerrada. Ficha Técnica 2: Nematología. IBTA-PROINPA. Cochabamba, Bolivia.

32. Otazú, V.; Brown, W. ; Quitón de, M. 1982. Enfermedades de las plantas en Bolivia. $30 \mathrm{p}$.

33. Peralta, L. 1996. Distribución e identificación de especies de Globodera spp. en las provincias de Yamparaez y Chayanta. Tesis 
Ing. Agr., Universidad Mayor Real y Pontificia de San Francisco Xavier de Chuquisaca. Sucre, Bolivia. $71 \mathrm{p}$.

34. Peralta, L; Franco, J.; Equize, H. 1995. Distribución e identificación de especies de Globodera spp. en las provincias de Yamparaez (Chuquisaca) y Chayanta (Potosí). En: Informe Anual IBTAPROINPA 1994-95. Cochabamba, Bolivia. p. 76 III.N.-78 III.N.

35. Ramos, J.; Franco, J.; Ortuño, N.; Oros, R.; Maín, G. 1998. Incidencia y severidad de Nacobbus aberrans y Globodera spp. en el cultivo de papa en Bolivia: Pérdidas en el valor bruto de su producción. IBTA-PROINPA. Cochabamba, Bolivia. 201 p.

36. Rivera, D.; Franco, J.; Montecinos, R.; Equize, H. 1993. Distribución, incidencia y hospedantes de Nacobbus aberrans. En: Informe Anual IBTA-PROINPA 1992-93. Cochabamba, Bolivia. p. 233 III.N.-237 III.N.

37. Rivera F., D. 1994. Incidencia, severidad y determinación de hospedantes de Nacobbus aberrans. Tesis Ing. Agr., Universidad de San Francisco Xavier. Sucre, Bolivia. 92 p.

38. Siles, E.; Oros, R.; Ortuño, N.; Franco, J. 1996. Distribución horizontal de Nacobbus aberrans en campos de agricultores. Pág. 73-74 En: Compendio de Exposiciones IV Reunión de la Papa. Octubre 8-11,1996. Cochabamba, Bolivia.

39. Villca, R. 1993. Evaluación de pérdidas causadas por Nacobbus aberrans en el cultivo de papa. Tesis Ing. Agr., Universidad Mayor Real y Pontificia de San Francisco Xavier de Chuquisaca. Sucre, Bolivia. 114p.

40. Tola M., A. 1997. Diagnóstico de suelos para la detección de Nacobbus aberrans y Globodera spp. en la zona de Araca (Prov. Loayza) La Paz. Tesis Ing. Agr., Universidad Mayor de San Andrés. La Paz, Bolivia. $131 \mathrm{p}$.

41. Torrico, W. 1988. Presencia de Nacobbus aberrans (Thorne y Allen) y Synchytrium endobioticum (Schilb.) Perc. en parcelas de productores de papa de la provincia Carrasco. Tesis Ing. Agr., Universidad Mayor de San Simón. Cochabamba, Bolivia. 122 p.

42. Zeballos, H. 1997. Aspectos económicos de la producción de papa en Bolivia. Informe al Centro Internacional de la Papa (CIP) y el Programa de Investigación de la Papa (PROINPA). 209 p. 